\title{
Selectivity of turned meshes codends for Deepwater Rose Shrimp (Parapenaeus longirostris), Horse Mackerel, (Trachurus trachurus) and European Hake (Merluccius merluccius) in the Aegean Sea
}

\section{Ege Denizi'nde Derinsu Pembe Karidesi (Parapenaeus longirostris), İstavrit (Trachurus trachurus) ve Bakalyaro için (Merluccius merluccius) döndürüllmüş ağ gözü torba seçiciliği}

\author{
1 İzmir Katip Çelebi University, Faculty of Fisheries, 35620, Çiğli, İzmir, Turkey \\ 2 Atatürk University, Faculty of Fisheries, 25240, Erzurum, Turkey \\ ${ }^{3}$ Ege University, Faculty of Fisheries, 35100, Bornova, İzmir, Turkey \\ * Corresponding author: sensurat@gmail.com
}

Tuğçe Şensurat Genç ${ }^{* *}$ (D) Muhammet Atamanalp2 (D) Celalettin Aydın ${ }^{3}$ (D)

How to cite this paper:

Şensurat Genç, T.Atamanalp, M. \& Aydın, C. (2018). Selectivity of turned meshes codends for Deepwater Rose Shrimp (Parapenaeus longirostris), Horse Mackerel, (Trachurus trachurus) and European Hake (Merluccius merluccius) in the Aegean Sea. Ege Journal of Fisheries and Aquatic Sciences, 35(2), 157168. DOI:10.12714/egejfas.2018.35.2.08

\begin{abstract}
More than fifty published on demersal trawl codend selectivity studies have been carried out in the Mediterranean waters of Turkey since the mid-1980s. In this study, in an attempt to enhance the size selectivity of three species in the Aegean Sea trawl fisheries, three $90^{\circ}$ turned meshes codens were investigated. Experiments were carried out in the Kuşadası Bay of the Eastern Mediterranean, between 19 January and 29 March 2015 with commercial stern trawler. The meshes of tested codends were turned 90, two of them have $44 \mathrm{~mm}$ mesh size with 300 and 150 meshes around codend circumferences (44T300 and 44T150). The other has $40 \mathrm{~mm}$ mesh size with 165 meshes around codend circumferences (40T165). Individual hauls and mean selectivity parameters were estimated by using the CC2000 and EC-Modeller softwares, respectively. The mean L50 values of 44T300, 44T150 and 40T165 were $17.5 \pm 0.2 \mathrm{~mm}, 18.6 \pm 0.1 \mathrm{~mm}$ and 17.1 $\pm 0.2 \mathrm{~mm}$ carapace length for shrimp, $16.4 \pm 0.4 \mathrm{~cm}, 17.1 \pm 0.4 \mathrm{~cm}$ and $14.8 \pm 0.2 \mathrm{~cm}$ total length for horse mackerel, respectively. The pooled $\mathrm{L}_{50}$ values of, 12.8 $\pm 0.5 \mathrm{~cm}, 13.2 \pm 0.6 \mathrm{~cm}$ and $12.1 \pm 0.1 \mathrm{~cm}$ total length for hake. While there are no differences between $\mathrm{L}_{50}$ values of $40 \mathrm{~T} 165$ and $44 \mathrm{~T} 300$ for deepwater rose shrimp ( $p>0.05)$, there are significant differences for horse mackerel $(p<0.05)$. The likelihood ratio test results showed that there were no significant differences among all codends for European hake. In conclusion, any effort such as using square mesh, turned mesh codends or halved the number of meshes in the codend to release immatures of some species, is probably to cause the loss of marketable sizes of other species in the Mediterranean. This economical loss of revenues is the essential concern of fishermen that prevents them using alternative codend designs, though such designs are technically applicable. Consequently, the losses of income caused by using T90 mesh codend and halving the number of meshes around codend circumference should also be investigated in future studies.
\end{abstract}

Keywords: Demersal trawl, turned meshes, deepwater rose shrimp, horse mackerel, European hake, Aegean Sea

Öz: Türkiye'nin Akdeniz sularında 1980'lerin ortalarından beri demersal trol torba seçiciliği üzerine elliden fazla çalışma yürütülmüştür. Bu çalışmada Ege Denizi trol balıkçılığında 3 türün boy seçiciliğini geliştirmek için 3 farklı döndürülmüş ağ göz torbaları araştııımıștır. Denemeler Doğu Akdeniz'de bulunan Kuşadası Körfezi'nde 19 Ocak ve 29 Mart 2015 tarihleri arasında ticari trol teknesiyle gerçekleştirilmiştir. Deneme torbalarının ağ gözleri $90^{\circ}$ döndürülmüş olup ikisi $44 \mathrm{~mm}$ ağ göz boyunda, 300 ve 150 torba çevre göz sayısına sahiptir (44T300 ve 44T150). Diğer torba 165 çevre göz sayısına sahip 40 mm ağ göz boyundadır. Bireysel çekimler ve ortalama seçicilik parametreleri sırasılla CC2000 ve EC-Modeller yaziıımları kullanılarak hesaplanmışıı. Derinsu pembe karidesinin 44T300, 44T150 ve 40T165 torbalarında ortalama $L_{50}$ değerleri sırasılyla $17,5 \pm 0,2 \mathrm{~mm}, 18,6 \pm 0,1 \mathrm{~mm}$ ve 17,1 $\pm 0,2 \mathrm{~mm}$ karapas boyu, istavritin $16,4 \pm 0,4 \mathrm{~cm}, 17,1 \pm 0,4 \mathrm{~cm}$ and $14,8 \pm 0,2 \mathrm{~cm}$ total boydur. Bakalyaronun birleștirimiş $L_{50}$ değerleri $12,8 \pm 0,5 \mathrm{~cm}, 13,2 \pm 0,6 \mathrm{~cm}$ ve $12,1 \pm 0,1 \mathrm{~cm}$ total boydur. Derinsu pembe karidesi için $40 T 165$ ve $44 T 300$ 'den elde edilen $L_{50}$ değerleri arasında fark yokken $(p>0,05)$, istavrit için önemli fark vardır $(p<0,05)$. Olabilirlik oran test sonuçları bakalyaro için tüm torbalar arasında istatistiksel olarak önemli bir fark bulunamamıştı. Sonuç olarak Akdenizde bazı türlerin jüvenillerini serbest bırakmak için kare gözlü, döndürülmüş gözlü torba kullanma veya torba etrafındaki göz sayısını yarıya düşürme gibi çabalar, pazarlanabilir boydaki diğer türlerin kaybına neden olabilmektedir. Alternatif torbalar teknik olarak uygulanabilir olmalarına rağmen, ekonomik gelir kaybı balıkçıların torbaları kullanmalarııı önleyen esas kaygıdır. Bu nedenle, T90 torba ve torba çevre göz sayııının yarıya indirilmesinin sebep olacağı gelir kayıpları gelecek çalışmalarda araştııımalıdır. 


\section{INTRODUCTION}

More than fifty scientific papers have been published on demersal trawl codend selectivity in the Mediterranean waters of Turkey since the mid-1980s (Stewart, 2002). The studies show that conventional mesh codends selectivity results are rather poor (Tokaç et al., 1998; Tosunoğlu et al., 2003; Tokaç et al., 2004, 2010; Özbilgin et al., 2012; Aydın et al., 2011). In order to improve selectivity, scientists have been made effort. One of these works is T90 mesh codends. T90 codend obtained by turning $90^{\circ}$ of standard diamond mesh and can create important improvements in size selectivity (Madsen, 2007; Wienbeck et al., 2011). T90 codend was first tested by Moderhak (1997) for cod (Gadus morhua) fishery and then scientific interest have been increasing all around the world (Arkley, 2008; Hansen, 2004; Herrmann et al., 2007; Wienbeck et al., 2011, 2014; Madsen et al., 2012; Gorman and Dixon, 2015; Herrmann et al., 2013; Madsen et al., 2015; Stepputtis et al., 2016). It has been introduced in 2006 the legislation (EU Regulation no. 2187/2005) for the Baltic Sea cod fishery (EU, 2005; Herrmann et al., 2007). On the otherhand, few studies have been conducted on selectivity of T90 codend in the Mediterranean. Kaykaç (2005) has investigated the selectivity of $40 \mathrm{~mm}$ turned meshes in Aegean Sea for Mullus barbatus, and Diplodus annularis. Tokaç et al. (2014) have compared the size selectivity of T0 and T90 codends with three different mesh sizes for Mullus barbatus, Pagellus erythrinus and Diplodus annularis. Aydın and Tokaç (2015) have compared the size selective properties of the same mesh size of $90^{\circ}$ turned mesh codend and square mesh codend for Parapenaeus longirostris and Phycis blennoides. Deval et al. (2016) estimated the selectivity of the same mesh size $(50 \mathrm{~mm})$ of T0 and T90 codends in deep water demersal trawl fisheries for $P$. longirostris, Plesionika martia, Aristaeomorpha foliacea and Aristeus antennatus. Dereli and Aydın (2016) gave selectivity results of four codends; two different mesh sizes of diamond mesh codends, square mesh codend and turned mesh codend for M. barbatus, Merluccius merluccius, Trachurus trachurus and Dentex moroccanus. Illkyaz et al. (2017) studied the selectivity of $90^{\circ}$ turned meshes of three different mesh size codends for bogue (Boops boops) in the Eastern Mediterranean.

Turkish Fishery Regulations (TFR) define a minimum mesh size of $44 \mathrm{~mm}$ diamond or $40 \mathrm{~mm}$ square mesh netting for demersal trawl codends used in Aegean Sea (Anonymous, 2016), However, Fishers widely use $40 \mathrm{~mm}$ diamond mesh netting in trawl codends, accept in practice as legal as it usually measures about $43 \mathrm{~mm}$ with a wedge gauge (Özbilgin et al. 2012). On the other hand, studies with the same mesh size codends have shown that the selectivity can be improved by reducing the meshes around the codens (Reeves et al., 1992; Broadhurst and Kennelly, 1996; Lök et al., 1997; Broadhurst et al., 2004; Özbilgin et al., 2005; Sala and Luchetti, 2011; Eryaşar et al., 2014). Since August 2013, there was the provision that "the number of meshes around codend can't be more than half of the number of meshes of tunnel" (Anonymous, 2012).
However, this provision is not present in the TFR which came into force in September 2016.

This study aimed to investigate the selectivity of $90^{\circ}$ turned meshes of $44 \mathrm{~mm}$ mesh sizes with 300 meshes around codend circumferences (44T300), $44 \mathrm{~mm}$ meshes size with 150 meshes around codend circumferences (44T150) and $40 \mathrm{~mm}$ mesh size with 165 meshes around codend circumferences (40T165) for the deepwater rose shrimp ( $P$. longirostris), horse mackerel ( $T$. trachurus) and European hake (M. merluccius) which have high economical value but different body shape. Selectivty results for hake firts time presented from the study.

\section{MATERIALS AND METHODS}

Experiments were carried out in the commercial fishing grounds of the Kuşadası Bay (Aegean Sea) during the period 19-24 January and 25-29 March 2015 on board a commercial stern trawler "Efsane G" (LAO: 19.85 m 500 HP engine power). A modified 900 meshes fishing circle demersal trawl net was used for all experiments (Şensurat, 2015). The water depth ranged from 65 to $215 \mathrm{~m}$ (average: $115.5 \mathrm{~m}$ ). The average towing duration was $178.1 \mathrm{~min}$. (110-250 min). All hauls were carried out during daytime.

All tested codends are same material ( $380 \mathrm{~d} / 21 \mathrm{no}$ ) made of knotted polyethylene netting and $5 \mathrm{~m}$ in length. The implementation of a T90 mesh where the diamond codend mesh (T0) is turned $90^{\circ}$ (Wienbeck et al., 2011). Characteristics of the experimental codends are as follows:

(1) 44T300: nominal $44 \mathrm{~mm}(45.4 \mathrm{~mm} \pm 0.11) 300$ meshes on its circumference.

(2) 44T150: nominal $44 \mathrm{~mm}(45.4 \mathrm{~mm} \pm 0.11), 150$ meshes on its circumference.

(3) 40T165: nominal $40 \mathrm{~mm}(40.4 \mathrm{~mm} \pm 0.10), 165$ meshes on its circumference.

The end of the tunnel consisted of $44 \mathrm{~mm}$ mesh size of 300 meshes in circumferences $(44 \times 300=13200 \mathrm{~mm})$. According to the EC regulation (Anonymous, 2006), the square mesh codend in particular, the circumference of the rear most part of the trawl body, and the extension piece should be from 2 to 4 times the circumference of the front end of the codend. We used the codends circumferences tested in the study considering the EC regulation as square mesh codend circumferences ((13200 mm / $40 \mathrm{~mm}) / 2=165$ meshes $)$ and $90^{\circ}$ turned meshes $((13200 \mathrm{~mm} / 40 \mathrm{~mm} / 2=165$ meshes $)$.

Full mesh sizes of the codends (four lines of 20 consecutive meshes in the towing direction) were measured while the netting was still wet after the tows by using OMEGA mesh gauge at $50 \mathrm{~N}$. Each codend was attached to end of the tunnel, which had 300 meshes in circumference made of $48 \mathrm{~mm}$ knotless PE netting. A protective bag, $5.5 \mathrm{~m}$ in length and knotted polypropilen material with $100 \mathrm{~mm}$ mesh size, was used around the codend whereby the protective bag was laced around the codend. Aft ends of the codend and the protective net were tied together. The main characteristic of the codends are given in Table 1. 
Table 1. Codend specifications

\begin{tabular}{lccc}
\hline Codend features & \multicolumn{3}{c}{ Tested Codends } \\
\hline & 44T300 & 44T150 & 40T165 \\
Nominal mesh size $(\mathrm{mm})$ & 44 & 44 & 40 \\
Measured mesh size $(\mathrm{mm})$ & 45.4 & 45.4 & 40.4 \\
Number of measurements & 80 & 80 & 80 \\
Twine thickness & $380 \mathrm{~d} / 21 \mathrm{no}$ & $380 \mathrm{~d} / 21 \mathrm{no}$ & $380 \mathrm{~d} / 21 \mathrm{no}$ \\
Materieal & PE knotted & PE knotted & PE knotted \\
Circumference mesh number & 300 & 150 & 165 \\
\hline
\end{tabular}

The selectivity of the codends was estimated using the hooped covered codend method described by Wileman et al. (1996). The cover was made of knotless polyamide (PA) netting with a nominal mesh size of $24 \mathrm{~mm}$ and supported by hoops, that $1.9 \mathrm{~m}$ in diameter. The hoops were made of $5 \mathrm{~cm}$ diameter PVC material. Supporting hoops were used to avoiding the masking effect of the cover on the codend meshes.

At the end of each tow the cover catch was first removed. If necessary, random sub-sampling of an appreciable amount for all hauls was made from the codend and the cover. The investigated species separately sorted from the rest of the catch and weighed. Meantime, crews sorted the marketable codend catch by species and left the discards on deck. Three species were deepwater rose shrimp, horse mackerel and hake, collected for selectivity analyses. Full or sub-samples were taken and weighed separately from marketable catch and discards.

Length measurements were obtained to the nearest $\mathrm{cm}$ for horse mackerel and European hake. Sub-samples were taken from the cover and codend for the deepwater rose shrimp. The length class frequencies were then estimated by raising the sub-sampled frequencies obtained by the ratio of the total weight to the sub-sample weight. The length class frequencies for the shrimps in the codend and cover were estimated by multiplying the measured frequencies in the subsamples by the inverse of the sampling proportion. The carapace length (from the orbital sinus to the internal posterior margin of the carapace) was measured to the nearest $\mathrm{mm}$ for the deep-water rose shrimp by using digital calipers. When the numbers of individuals retained and escaped were insufficient for individual haul estimation, data were pooled over all hauls to estimate the selection curve for only European hake. The length distributions of the discarded individuals were added to the codend's distribution for the selectivity analysis.

To compare the retention rates of immature specimens between the different codend, a 13 and $20 \mathrm{~cm}$ total length (TL) minimum landing sizes (MLS) were used for Atlantic horse mackerel and European hake given by Anonymous (2016), respectively. MLS regulation does not given in TFR for deepwater rose shrimp. Therefore, $20 \mathrm{~mm}$ carapace length (CL) MLS were used for rose shrimp given by EU (2005).
Selectivity parameters for individual hauls were obtained by using the CC2000 software (ConStat, 1995). The data were analized using a logistic equation with the maximum likelihood method (Wileman et al., 1996) as $\mathrm{S}(\mathrm{l})=$ $\exp (\mathrm{v} 1+\mathrm{v} 2 \mathrm{l}) /[1+\exp (\mathrm{v} 1+\mathrm{v} 2 \mathrm{l})], \quad$ where the parameters $v_{1}$ and $v_{2}$ are the intercept and slope of the linear logistic function, respectively. The mean selectivity of the individual hauls was calculated by taking into account betweenhaul variation according to Fryer (1991) using the ECModeller software (ConStat, 1995) which adopts the REML method (residual maximum likelihood). The values of $L_{50}$ were estimated from the expression:

$$
L 50=(-v 1 / v 2)
$$

Selection Range (SR, difference between the $75 \%$ and $25 \%$ retention lengths) was estimated with the equation:

$$
S R=L 75-L 25
$$

Fryer (1991) model was used to test between-haul variation of the selectivity parameters $v_{1}$ and $v_{2}$ by mesh configuration, allowing the estimation of mean curves for the three different mesh codends by using the software ECModeller (ConStat, 1995) which utilizes the REML (Residual Maximum Likelihood) approach. The model of Fryer (1991) was also used to investigate the significance of catch size, mesh configuration on the selectivity parameter estimates. Some of the explanatory variables effect on the $L_{50}$ and $S R$ values such as the mesh configuration, mesh numbers of codend circumference, the codend catch, the species catch and the haul duration were tested.

The statistical significance among pooled selection curves for the European hake was evaluated with a likelihood ratio test (e.g. Campos et al. 2003).

\section{RESULTS}

A total weight of 5.02 tonnes was caught in the codend and cover during $100.9 \mathrm{~h}$, in 34 hauls (11 hauls 44T300, 11 hauls 44T150 and 12 hauls 40T165). The total catch was composed of $0.93 \mathrm{t}$ with $44 \mathrm{~T} 300,2.36 \mathrm{t}$ with $44 \mathrm{~T} 150$ and $1.73 \mathrm{t}$ with 40T165. Deepwater rose shrimp (P. longirostris), Horse mackerel ( $T$. trachurus) and European hake (M. merluccius) accounted for the majority of the total catch by weight (Table 2). 
Table 2. The total catch and percentage of catch by species in codend and cover as caught from 44T300, 44T150 and 40T165 codends

\begin{tabular}{lccccccccc} 
& & $\mathbf{4 4 T 3 0 0}$ & \multicolumn{3}{c}{ 44T150 } & \multicolumn{3}{c}{$\mathbf{4 0 T 1 6 5}$} \\
& Codend & Cover & Total & Codend & Cover & Total & Codend & Cover & Total \\
\hline Weight $(\mathbf{k g})$ & 750.5 & 182.2 & 932.7 & 2138.0 & 219.3 & 2357.3 & 1398.4 & 336.4 & 1734.8 \\
Deepwater rose shrimp $(\%)$ & 5.9 & 16.5 & 7.9 & 0.6 & 5.3 & 1.0 & 3.3 & 9.0 & 4.6 \\
Horse mackerel $(\%)$ & 8.7 & 16.8 & 10.2 & 3.1 & 19.9 & 4.6 & 20.1 & 30.9 & 23.5 \\
European hake (\%) & 11.1 & 0 & 8.9 & 2.5 & 0.2 & 2.3 & 4.0 & 0.1 & 3.2 \\
Others $(\%)$ & 74.3 & 66.7 & 73.0 & 93.8 & 74.6 & 92.1 & 72.6 & 60.0 & 68.7 \\
\hline
\end{tabular}

\section{Deepwater rose shrimp (Parapenaeus longirostris Lucas, 1846)}

In total, 51276 deepwater rose shrimp were caught from 33 valid hauls. While $52.4 \%$ (26 888) specimens retained, $47.6 \%$ (24 388) was escaped.
Total number of specimens, captured and escaped percentage and their minimum and maximum sizes in codends are given in Table 3. Length-frequency distributions show their size ranges between 7 to $32 \mathrm{~mm}$ for all tested codends, with a main peak at 15-17 mm CL (Figure 1, right side). A total of 43.6, 56.1 and $32.9 \%$ of the specimens were found above MLS in the 44T300, 44T150 and 40T165 codend, respectively.

Table 3. Total number of deepwater rose shrimp specimens, captured and escaped percentage and their minimum and maximum sizes in codends

\begin{tabular}{|c|c|c|c|c|c|c|c|c|c|}
\hline & \multirow{2}{*}{$\begin{array}{c}\text { Total } \\
\text { number of } \\
\text { specimens }\end{array}$} & \multirow{2}{*}{$\begin{array}{c}\text { Captured } \\
(\%)\end{array}$} & \multicolumn{2}{|c|}{ Size ranges $(\mathrm{mm})$} & \multirow{2}{*}{$\begin{array}{c}\text { Above } \\
\text { MLS (\%) }\end{array}$} & \multirow{2}{*}{$\begin{array}{c}\text { Escaped } \\
(\%)\end{array}$} & \multicolumn{2}{|c|}{ Size ranges $(\mathrm{mm})$} & \multirow{2}{*}{$\begin{array}{c}\text { Above MLS } \\
(\%)\end{array}$} \\
\hline & & & Min & $\operatorname{Max}$ & & & Min & $\operatorname{Max}$ & \\
\hline $44 T 300$ & 21333 & 54.7 & 7 & 32 & 43.6 & 45.3 & 6 & 27 & 12.6 \\
\hline $44 \mathrm{~T} 150$ & 7030 & 43.4 & 10 & 32 & 56.1 & 56.6 & 7 & 27 & 15.6 \\
\hline $40 \mathrm{~T} 165$ & 22913 & 53.1 & 9 & 31 & 32.9 & 46.9 & 8 & 28 & 13.6 \\
\hline
\end{tabular}

Selectivity estimates for individuals and mean curves (according to Fryer, 1991) of rose shrimp are given in Table 4 and Figure 1. Table 4 also presents numbers of shrimp in codends and covers. The mean $L_{50}$ and $S R$ values were 17.5 and $5.7 \mathrm{~mm}$ for $44 \mathrm{~T} 300,18.6$ and $5.1 \mathrm{~mm}$ for $44 \mathrm{~T} 150$ and 17.1 and $6.0 \mathrm{~mm}$ for 40T165. An inspectation of the fit statistics indicated problems using a logistic curve to describe the selection data for all hauls ( $p<0.05)$, except for hauls no. 3, 4, 7 and 10 for 44T300, no. 1, 2, 3, 7, 10, 11 for 44T150 and no. $4,5,6,7$ and 10 for 44T165. Inspection of the deviance residuals indicated structural problems when using the logistic curve to model the experimental data in these hauls. Lack of fit does not certainly refer that the fitted selection curve is not a good model of the selection of the species. If a plot of residuals vs. length shows no clear structure, then the lack of fit is due to over-dispersion (McCullagh and Nelder, 1989; Deval et al., 2016), that is, the failure of the assumption that fish behave independently (Wileman et al., 1996).
Significant differences were found only between $44 \mathrm{~T} 150$ and the other two codends. On the other hand, there were no significant differences between SR values between the codends $(p>0.05)$. Explanatory variables results which affect selectivity parameters are also given in Table 5.

\section{Horse mackerel (Trachurus trachurus Linnaeus, 1758)}

A total number of 13562 horse mackerel were caught with 30 valid hauls. While $63.5 \%$ (8617) specimens retained, $\% 36.5$ (4945) was escaped. Total number of specimens, captured and escaped percentage and their minimum and maximum sizes in codends are given in Table 6. Length-frequency distributions show their size ranges as 12.5 to $31 \mathrm{~cm}$ for all tested codends. All of the captured specimens in the 44T300 and 44T150 codends, $99.7 \%$ of the specimens were found above MLS in 40T165 codend (13 cm, Anonymus, 2016). 

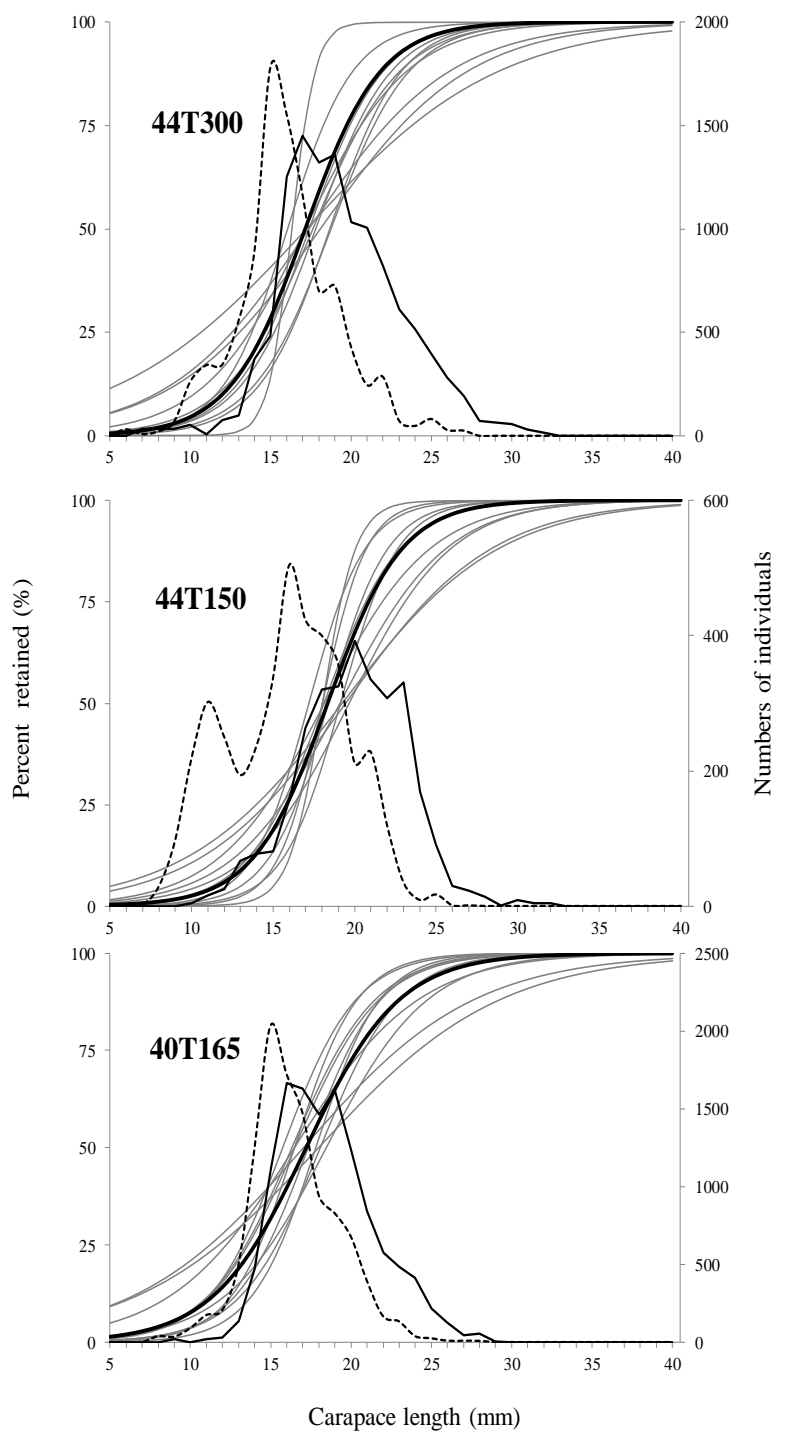

Figure 1. The selection curves and length distribution of the deepwater rose shrimp ( $P$. longirostris). Y-axis left: percentage retained for the selection curves of: 44T300, 44T150 and 40T165 (thick drawn lines; mean selection curve (Fryer 1991), thin drawn lines; individual selection curves). Y-axis right: normalized length-frequency distribution, drawn lines: codend specimens, broken lines; and cover specimens.
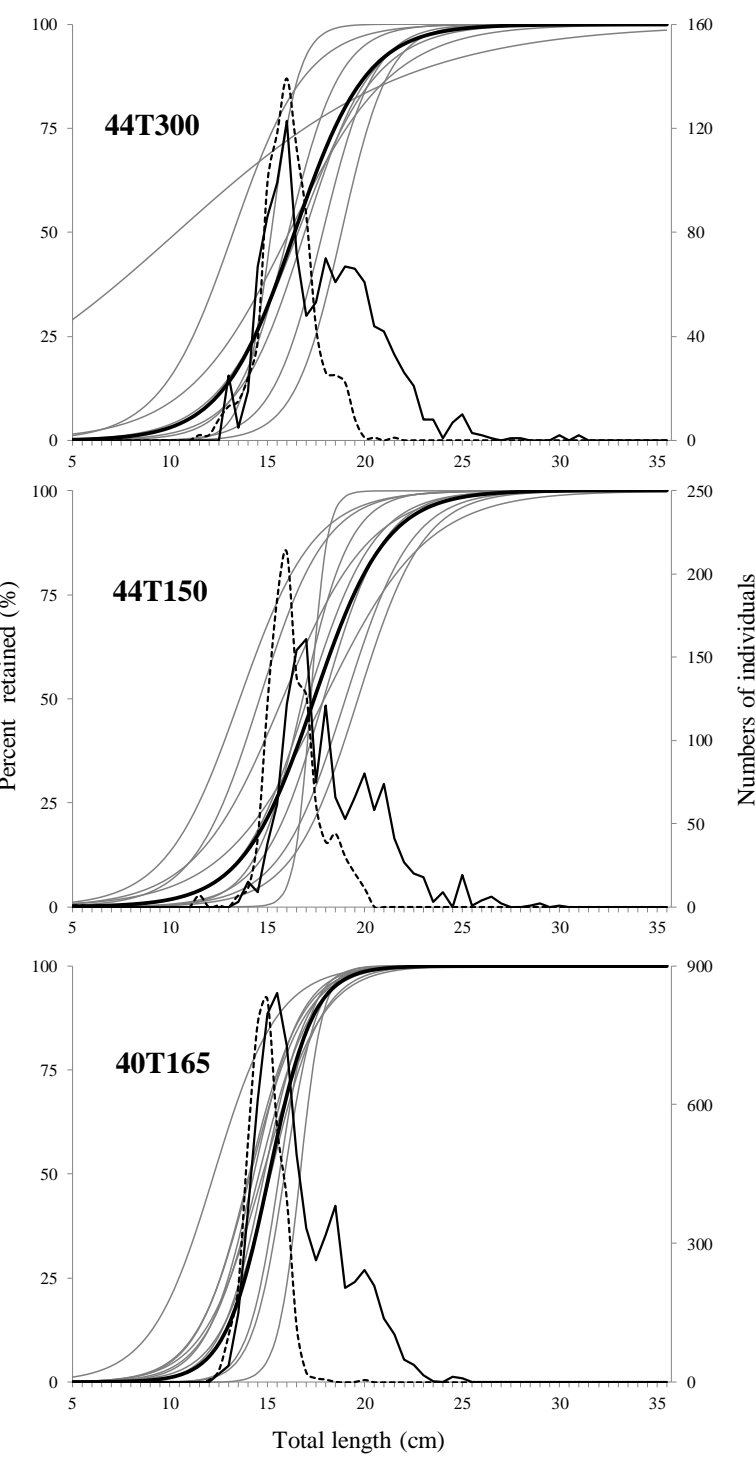

Figure 2. The selection curves and length distribution of the horse mackerel ( $T$. trachurus). Y-axis left: percentage retained for the selection curves of: 44T300, 44T150 and 40T165 (thick drawn lines; mean selection curve (Fryer 1991), thin drawn lines; individual selection curves). Y-axis right: normalized length-frequency distribution, drawn lines: codend specimens, broken lines; and cover specimens

Table 4. Estimated selectivity parameters of individual and mean hauls (according to Fryer, 1991) for deepwater rose shrimp in 44T300, 44T150 and $40 \mathrm{~T} 165$

\begin{tabular}{|c|c|c|c|c|c|c|c|c|c|c|c|c|c|c|c|}
\hline Codends & H.N & L50 & $\begin{array}{l}\text { Cl low- } \\
\mathrm{Cl} \text { high }\end{array}$ & SR & $\begin{array}{l}\text { Cl low-Cl } \\
\text { high }\end{array}$ & $\mathrm{v}_{1}$ & $\mathbf{v}_{2}$ & $\mathbf{R}_{11}$ & $\mathbf{R}_{12}$ & $\mathbf{R}_{22}$ & deviance & dof & pvalue & $\mathrm{NCd}$ & $\mathrm{NCv}$ \\
\hline \multirow{9}{*}{ 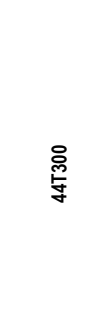 } & 1 & 18.7 & $18.0-19.5$ & 5.3 & $3.8-6.9$ & -7.707 & 0.411 & 1.061 & -0.057 & 0.003 & 96.22 & 14 & 0.00 & 914 & 1035 \\
\hline & 2 & 17.6 & $16.8-18.5$ & 5.9 & $3.9-8.0$ & -6.525 & 0.370 & 1.222 & -0.067 & 0.004 & 143.99 & 21 & 0.00 & 820 & 667 \\
\hline & 3 & 16.1 & $15.5-16.7$ & 4.5 & $3.1-5.8$ & -7.930 & 0.494 & 1.209 & -0.075 & 0.005 & 22.11 & 13 & 0.05 & 133 & 143 \\
\hline & 4 & 18.7 & $17.7-19.7$ & 4.9 & $3.1-6.7$ & -8.388 & 0.449 & 2.416 & -0.124 & 0.007 & 15.14 & 18 & 0.65 & 79 & 64 \\
\hline & 5 & 17.2 & $15.5-18.9$ & 13.1 & $5.6-20.7$ & -2.878 & 0.167 & 0.645 & -0.037 & 0.002 & 1070.21 & 23 & 0.00 & 5083 & 5146 \\
\hline & 6 & 16.3 & $16.0-16.6$ & 1.6 & $1.3-1.9$ & -22.377 & 1.372 & 4.854 & -0.286 & 0.017 & 97.01 & 23 & 0.00 & 2371 & 896 \\
\hline & 7 & 17.1 & $16.3-18.0$ & 6.9 & $4.8-9.0$ & -5.441 & 0.317 & 0.689 & -0.038 & 0.002 & 11.74 & 19 & 0.90 & 294 & 144 \\
\hline & 8 & 17.4 & $16.5-18.2$ & 9.6 & $7.0-12.1$ & -3.980 & 0.229 & 0.286 & -0.015 & 0.001 & 88.81 & 21 & 0.00 & 1155 & 999 \\
\hline & 9 & 17.4 & $16.6-18.2$ & 5.1 & $3.8-6.3$ & -7.568 & 0.435 & 0.876 & -0.047 & 0.003 & 59.54 & 20 & 0.00 & 469 & 325 \\
\hline
\end{tabular}




\begin{tabular}{|c|c|c|c|c|c|c|c|c|c|c|c|c|c|c|c|}
\hline & 10 & 17.8 & 16.5-19.1 & 5.2 & $3.2-7.1$ & -7.567 & 0.425 & 2.212 & -0.113 & 0.006 & 12.17 & 18 & 0.84 & 94 & 40 \\
\hline & 11 & 18.1 & $15.9-20.3$ & 10.1 & $4.2-15.9$ & -3.944 & 0.218 & 1.341 & -0.068 & 0.004 & 79.88 & 17 & 0.00 & 258 & 204 \\
\hline & $F$ & 17.5 & $17.3-17.7$ & 5.7 & $5.3-6.1$ & -7.309 & 0.426 & 21.479 & -1.337 & 0.084 & 17.00 & 17 & 0.00 & & \\
\hline \multirow{12}{*}{$\frac{\text { R }}{\frac{8}{8}}$} & 1 & 18.1 & $17.2-19.0$ & 5.1 & $3.1-7.1$ & -7.768 & 0.429 & 2.086 & -0.114 & 0.006 & 16.3 & 17 & 0.50 & 76 & 80 \\
\hline & 2 & 17.2 & $15.9-18.5$ & 3.8 & $2.1-5.4$ & -9.988 & 0.581 & 4.948 & -0.262 & 0.014 & 10.9 & 14 & 0.69 & 84 & 23 \\
\hline & 3 & 18.0 & $16.1-19.9$ & 3.0 & $0.1-5.9$ & -13.369 & 0.742 & 32.045 & -1.736 & 0.095 & 7.2 & 7 & 0.41 & 11 & 10 \\
\hline & 4 & 18.2 & $16.9-19.5$ & 7.1 & $3.8-10.4$ & -5.587 & 0.308 & 1.517 & -0.081 & 0.005 & 109.4 & 15 & 0.00 & 511 & 513 \\
\hline & 5 & 18.4 & $17.9-18.9$ & 4.0 & $3.1-4.9$ & -10.095 & 0.549 & 1.258 & -0.065 & 0.003 & 48.9 & 18 & 0.00 & 705 & 414 \\
\hline & 6 & 19.1 & $18.2-20.1$ & 7.2 & $4.9-9.5$ & -5.685 & 0.307 & 0.784 & -0.041 & 0.002 & 93.7 & 19 & 0.00 & 603 & 753 \\
\hline & 7 & 19.1 & $18.5-19.8$ & 3.9 & $2.7-5.0$ & -10.904 & 0.570 & 2.428 & -0.129 & 0.007 & 14.2 & 20 & 0.82 & 85 & 134 \\
\hline & 8 & 19.7 & $18.4-20.9$ & 6.9 & $4.4-9.4$ & -6.293 & 0.320 & 0.989 & -0.054 & 0.003 & 166.8 & 17 & 0.00 & 474 & 1020 \\
\hline & 9 & 19.2 & $16.9-21.5$ & 10.5 & $5.2-15.9$ & -4.002 & 0.208 & 0.728 & -0.042 & 0.003 & 145.9 & 19 & 0.00 & 387 & 739 \\
\hline & 10 & 18.0 & $17.4-18.7$ & 2.2 & $1.5-3.0$ & -17.775 & 0.986 & 6.822 & -0.403 & 0.024 & 10.6 & 17 & 0.88 & 48 & 203 \\
\hline & 11 & 19.4 & $17.7-21.1$ & 9.8 & $5.5-14.1$ & -4.354 & 0.224 & 0.804 & -0.041 & 0.002 & 14.8 & 18 & 0.68 & 70 & 84 \\
\hline & $F$ & 18.6 & $18.5-18.7$ & 5.1 & $4.7-5.5$ & -8.040 & 0.438 & 9.165 & -0.488 & 0.026 & 17.00 & 17 & 0.00 & & \\
\hline \multirow{11}{*}{ 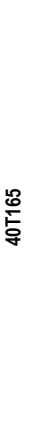 } & 1 & 16.5 & $15.8-17.2$ & 8.5 & $6.1-10.9$ & -4.266 & 0.259 & 0.352 & -0.020 & 0.001 & 329.9 & 19 & 0.00 & 5487 & 4697 \\
\hline & 2 & 18.0 & $17.0-19.0$ & 12.4 & $7.9-17.0$ & -3.181 & 0.177 & 0.295 & -0.016 & 0.001 & 119.8 & 17 & 0.00 & 2014 & 2142 \\
\hline & 3 & 18.5 & $17.7-19.3$ & 6.9 & $4.7-9.1$ & -5.844 & 0.317 & 0.738 & -0.040 & 0.002 & 98.6 & 17 & 0.00 & 927 & 1088 \\
\hline & 4 & 18.1 & $17.7-18.5$ & 5.4 & $4.4-6.5$ & -7.303 & 0.404 & 0.467 & -0.025 & 0.001 & 23.2 & 15 & 0.08 & 391 & 346 \\
\hline & 5 & 16.5 & $16.1-16.8$ & 4.3 & $3.6-5.0$ & -8.398 & 0.510 & 0.475 & -0.027 & 0.002 & 24.2 & 15 & 0.06 & 634 & 331 \\
\hline & 6 & 17.3 & $16.0-18.6$ & 5.0 & $2.1-7.9$ & -7.640 & 0.441 & 4.499 & -0.253 & 0.014 & 8.3 & 13 & 0.83 & 40 & 37 \\
\hline & 7 & 15.7 & $14.9-16.6$ & 5.0 & $3.7-6.3$ & -6.876 & 0.437 & 0.688 & -0.044 & 0.003 & 23.0 & 16 & 0.11 & 110 & 136 \\
\hline & 8 & 17.0 & $15.7-18.4$ & 11.6 & $6.1-17.2$ & -3.217 & 0.189 & 0.603 & -0.033 & 0.002 & 83.3 & 18 & 0.00 & 779 & 655 \\
\hline & 9 & 16.3 & $15.8-16.9$ & 5.7 & $4.3-7.1$ & -6.280 & 0.385 & 0.553 & -0.032 & 0.002 & 85.0 & 18 & 0.00 & 1417 & 1053 \\
\hline & 10 & 17.8 & $16.8-18.8$ & 4.4 & $2.6-6.2$ & -8.915 & 0.501 & 3.061 & -0.169 & 0.010 & 10.0 & 15 & 0.82 & 60 & 56 \\
\hline & $\begin{array}{l}11 \\
F\end{array}$ & $\begin{array}{l}16.5 \\
17.1\end{array}$ & $\begin{array}{l}15.5-17.5 \\
16.9-17.3\end{array}$ & $\begin{array}{l}5.8 \\
6.0\end{array}$ & $\begin{array}{l}3.8-7.7 \\
5.7-6.3\end{array}$ & $\begin{array}{l}-6.263 \\
-5.938\end{array}$ & $\begin{array}{l}0.380 \\
0.345\end{array}$ & $\begin{array}{l}1.121 \\
2.915\end{array}$ & $\begin{array}{l}-0.063 \\
-0.166\end{array}$ & $\begin{array}{l}0.004 \\
0.010\end{array}$ & $\begin{array}{c}51.2 \\
17\end{array}$ & $\begin{array}{l}18 \\
17\end{array}$ & $\begin{array}{l}0.00 \\
0.00\end{array}$ & 305 & 208 \\
\hline
\end{tabular}

L50: 50\% retention length (mm); Cl: confidence interval; SR: selection range; $\mathrm{v}_{1}$ and $\mathrm{v}_{2}$ : regression parameters; dof: degree of freedom; R: variance matrix measuring the within-haul variation; NCd: Number of specimens in Codend; NCv: Number of specimens in Cover

Table 5. Explanatory variables affected on selectivity parameters

\begin{tabular}{llllll}
\hline Parameters & Estimate & SD & t-value & dof & p-value \\
\hline P. longirostris & & & & & \\
Mesh (L50) & -16.053 & 1.418 & -11.331 & 35 & 0.000 \\
Mesh (SR) & -4.565 & 1.543 & -2.958 & 35 & 0.006 \\
Codend species catch (L50) & -0.000 & 0.000 & -2.317 & 35 & 0.027 \\
Duration (L50) & 0.020 & 0.007 & 2.777 & 35 & 0.009 \\
Circumference (L50) & 15.293 & 1.224 & 12.492 & 35 & 0.000 \\
Circumference (SR) & 5.142 & 0.691 & 7.442 & 35 & 0.000 \\
\hline
\end{tabular}

L50: $50 \%$ retention length, SR: selection range, SD: standart deviation; dof: degree of freedom

Table 6. Total number of horse mackerel specimens, captured and escaped percentage and their minimum and maximum sizes in codends

\begin{tabular}{|c|c|c|c|c|c|c|c|c|c|}
\hline & \multirow{2}{*}{$\begin{array}{c}\text { Total } \\
\text { number of } \\
\text { specimens }\end{array}$} & \multirow{2}{*}{$\begin{array}{c}\text { Captured } \\
(\%)\end{array}$} & \multicolumn{2}{|c|}{ Size ranges $(\mathrm{mm})$} & \multirow{2}{*}{$\begin{array}{l}\text { Above } \\
\text { MLS (\%) }\end{array}$} & \multirow{2}{*}{$\begin{array}{c}\text { Escaped } \\
(\%)\end{array}$} & \multicolumn{2}{|c|}{ Size ranges $(\mathrm{mm})$} & \multirow{2}{*}{$\begin{array}{c}\text { Above MLS } \\
(\%)\end{array}$} \\
\hline & & & Min & $\operatorname{Max}$ & & & Min & $\operatorname{Max}$ & \\
\hline 44T300 & 1365 & 67.4 & 13.5 & 31.0 & 100 & 32.6 & 11.5 & 21.5 & 98.0 \\
\hline $44 \mathrm{~T} 150$ & 2367 & 55.3 & 13.5 & 30.0 & 100 & 44.7 & 5 & 20 & 99.1 \\
\hline 40T165 & 9830 & 65.0 & 12.5 & 25.0 & 99.7 & 35.0 & 10 & 20 & 99.2 \\
\hline
\end{tabular}

Thirty valid hauls provided data to obtain selection curves for all codends. Selectivity estimates for individuals and mean curves (according to Fryer, 1991) of horse mackerel are given in Table 7 and Figure 2. The mean $L_{50}$ values for $44 T 300$, 44T150 and 40T165 were 16.4, 17.1 and $14.8 \mathrm{~cm}$, respectively. SR values of these three codends were $2.7,3.4$ and $2.5 \mathrm{~cm}$, respectively. Inspection of the fit statistics ( $p$-value and model deviance vs. df) indicated that there were no problems using a logit curve to describe the selection data for any haul $(p>0.05)$, except for hauls no. 5, 6 and 10 for 44T150, and no. 1, 2, 4, 5, $6,8,9$ and 11 for $44 \mathrm{~T} 165$. There were significant differences between $L_{50}$ values of $40 T 165$ and the other two codends $(p<$ 0.05). Explanatory variables which affect on selectivity parameters are given in Table 8. 
Table 7. Estimated selectivity parameters of individual and mean hauls (according to Fryer, 1991) for horse mackerel in 44T300, 44T150 and 40T165

\begin{tabular}{|c|c|c|c|c|c|c|c|c|c|c|c|c|c|c|c|}
\hline Codends & H.N & $\mathrm{L}_{50}$ & $\begin{array}{l}\text { Cl low- } \\
\mathrm{Cl} \text { high }\end{array}$ & SR & $\begin{array}{l}\text { Cl low- } \\
\mathrm{Cl} \text { high }\end{array}$ & $v_{1}$ & $\mathbf{V}_{2}$ & $\mathbf{R}_{11}$ & $\mathbf{R}_{12}$ & $\mathbf{R}_{22}$ & deviance & dof & $\begin{array}{c}\mathrm{p} \\
\text { value }\end{array}$ & $\mathrm{NCd}$ & $\mathrm{NCv}$ \\
\hline \multirow{10}{*}{$\begin{array}{l}\text { 足 } \\
\text { 年 }\end{array}$} & 1 & 15.2 & $14.5-16.0$ & 1.6 & $0.7-2.5$ & -21.271 & 1.398 & 37.867 & -2.344 & 0.146 & 7.08 & 18 & 0.99 & 170 & 11 \\
\hline & 2 & 16.9 & $14.7-19.2$ & 3.7 & $0.4-7.1$ & -0.954 & 0.588 & 14.070 & -0.907 & 0.059 & 11.91 & 14 & 0.61 & 8 & 24 \\
\hline & 3 & 16.1 & $12.8-19.5$ & 2.9 & $-1.1-7.0$ & -12.044 & 0.748 & 41.922 & -2.774 & 0.187 & 5.51 & 7 & 0.60 & 3 & 9 \\
\hline & 4 & 16.6 & $15.6-17.6$ & 4.5 & $2.7-6.4$ & -8.077 & 0.487 & 2.971 & -0.164 & 0.009 & 19.96 & 23 & 0.65 & 113 & 49 \\
\hline & 5 & 13.3 & $10.7-15.8$ & 4.1 & $1.6-6.6$ & -7.042 & 0.531 & 7.020 & -0.405 & 0.024 & 14.59 & 21 & 0.84 & 192 & 16 \\
\hline & 6 & 10.4 & $5.1-15.6$ & 13.2 & $0.9-25.4$ & -1.731 & 0.167 & 1.326 & -0.083 & 0.005 & 22.77 & 14 & 0.06 & 316 & 124 \\
\hline & 7 & 18.8 & $18.1-19.5$ & 2.7 & $1.8-3.6$ & -15.486 & 0.823 & 5.361 & -0.304 & 0.017 & 24.01 & 15 & 0.07 & 48 & 159 \\
\hline & 8 & 17.9 & $17.2-18.5$ & 2.9 & $1.7-4.1$ & -13.553 & 0.759 & 7.511 & -0.419 & 0.024 & 10.46 & 25 & 1.00 & 62 & 46 \\
\hline & 9 & 16.1 & $12.6-19.7$ & 5.8 & $-7.9-19.6$ & -6.066 & 0.376 & 34.868 & -2.134 & 0.132 & 6.71 & 6 & 0.35 & 8 & 7 \\
\hline & $F$ & 16.4 & $16.0-16.8$ & 2.7 & $2.5-2.9$ & -8.785 & 0.536 & 16.607 & -0.779 & 0.038 & & 13 & 0.00 & & \\
\hline \multirow{11}{*}{ 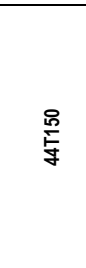 } & 1 & 17.2 & 14.6-19.9 & 0.9 & $-0.9-2.7$ & -41.512 & 2.407 & 1005.21 & -62.892 & 3.938 & 3.07 & 7 & 0.88 & 1 & 55 \\
\hline & 2 & 19.0 & 18.3-19.7 & 3.7 & $2.8-4.7$ & -11.205 & 0.589 & 1.503 & -0.088 & 0.005 & 32.44 & 25 & 0.15 & 112 & 341 \\
\hline & 3 & 17.9 & $17.3-18.5$ & 3.4 & $2.2-4.7$ & -11.457 & 0.640 & 3.570 & -0.204 & 0.012 & 15.82 & 18 & 0.61 & 81 & 107 \\
\hline & 4 & 15.8 & $14.9-16.7$ & 4.9 & $2.4-7.5$ & -7.011 & 0.444 & 3.288 & -0.195 & 0.012 & 17.43 & 16 & 0.36 & 134 & 79 \\
\hline & 5 & 19.6 & $18.6-20.7$ & 3.7 & $2.2-5.2$ & -11.661 & 0.593 & 4.183 & -0.233 & 0.013 & 32.52 & 20 & 0.04 & 69 & 234 \\
\hline & 6 & 13.6 & $11.7-15.5$ & 4.2 & $1.7-6.6$ & -7.138 & 0.525 & 5.993 & -0.359 & 0.022 & 47.16 & 18 & 0.00 & 510 & 81 \\
\hline & 7 & 14.6 & $13.3-16.0$ & 3.7 & $2.0-5.4$ & -8.623 & 0.590 & 4.903 & -0.284 & 0.017 & 29.06 & 18 & 0.05 & 324 & 54 \\
\hline & 8 & 17.9 & $16.6-19.2$ & 5.8 & $2.1-9.5$ & -6.779 & 0.378 & 3.897 & -0.226 & 0.013 & 11.37 & 17 & 0.84 & 47 & 63 \\
\hline & 9 & 17.1 & $15.7-18.5$ & 3.8 & $0.4-7.1$ & -9.917 & 0.581 & 15.573 & -0.947 & 0.058 & 9.86 & 15 & 0.83 & 22 & 26 \\
\hline & 10 & 16.8 & 14.3-19.4 & 2.7 & $-2.3-7.7$ & -12.682 & 0.813 & 102.155 & -6.385 & 0.401 & 15.25 & 7 & 0.03 & 9 & 18 \\
\hline & $F$ & 17.1 & $16.7-17.5$ & 3.4 & $3.2-3.6$ & -9.415 & 0.541 & 2.862 & -0.055 & 0.001 & & 15 & 0.00 & & \\
\hline \multirow{12}{*}{ 吕 } & 1 & 14.2 & 13.6-14.7 & 3.1 & $2.1-4.1$ & -10.050 & 0.710 & 2.809 & -0.181 & 0.012 & 43.0 & 16 & 0.00 & 953 & 288 \\
\hline & 2 & 15.6 & $15.1-16.1$ & 1.8 & $0.1-2.5$ & -19.352 & 1.238 & 12.648 & -0.811 & 0.052 & 155.7 & 19 & 0.00 & 685 & 375 \\
\hline & 3 & 15.9 & $15.6-16.2$ & 1.8 & $1.3-2.4$ & -19.178 & 1.206 & 8.137 & -0.511 & 0.032 & 18.7 & 18 & 0.41 & 150 & 90 \\
\hline & 4 & 16.7 & $16.4-17.0$ & 1.3 & $0.9-1.6$ & -28.753 & 1.722 & 13.927 & -0.866 & 0.054 & 47.3 & 19 & 0.00 & 188 & 524 \\
\hline & 5 & 15.2 & $14.7-15.7$ & 2.9 & $1.4-4.4$ & -11.625 & 0.765 & 7.101 & -0.476 & 0.032 & 56.0 & 11 & 0.00 & 385 & 496 \\
\hline & 6 & 14.9 & 14.4-15.3 & 2.5 & $1.4-3.6$ & -13.239 & 0.891 & 8.214 & -0.534 & 0.035 & 33.6 & 13 & 0.00 & 272 & 155 \\
\hline & 7 & 14.8 & $14.3-15.3$ & 3.4 & $2.1-4.6$ & -9.667 & 0.654 & 3.014 & -0.197 & 0.013 & 20.4 & 16 & 0.20 & 143 & 87 \\
\hline & 8 & 14.6 & $14.0-15.1$ & 3.0 & $1.8-4.1$ & -10.855 & 0.744 & 4.616 & -0.297 & 0.019 & 71.7 & 14 & 0.00 & 1033 & 406 \\
\hline & 9 & 14.3 & $13.9-14.8$ & 2.6 & $1.7-3.6$ & -11.961 & 0.835 & 4.654 & -0.309 & 0.021 & 97.4 & 14 & 0.00 & 1374 & 619 \\
\hline & 10 & 12.3 & $10.3-14.2$ & 3.6 & $1.3-5.9$ & -7.491 & 0.610 & 7.513 & -0.495 & 0.033 & 15.3 & 13 & 0.29 & 230 & 33 \\
\hline & 11 & 14.1 & $13.6-14.6$ & 3.0 & $1.9-4.0$ & -10.421 & 0.740 & 3.600 & -0.239 & 0.016 & 76.7 & 19 & 0.00 & 975 & 369 \\
\hline & $F$ & 14.8 & $14.6-15.0$ & 2.5 & $2.4-2.6$ & -13.328 & 0.793 & 30.492 & -1.628 & 0.087 & & 17 & 0.00 & & \\
\hline
\end{tabular}

$\mathrm{L}_{50}$ : $50 \%$ retention length $(\mathrm{mm})$; Cl: confidence interval; SR: selection range; $\mathrm{v}_{1}$ and $\mathrm{v}_{2}$ : regression parameters; dof: degree of freedom; R: variance matrix measuring the within-haul variation; NCd: Number of specimens in Codend; NCv: Number of specimens in Cover

Table 8. Explanatory variables affected on selectivity parameters

\begin{tabular}{llllll}
\hline Alpha parameters & Estimate & SD & t-value & dof & p-value \\
\hline T. trachurus & & & & & \\
Codend catch $(S R)$ & 0.000 & 0.000 & 10.661 & 32 & 0.000 \\
Codend species catch $\left(L_{50}\right)$ & -0.000 & 0.000 & -2.230 & 32 & 0.033 \\
Duration $\left(L_{50}\right)$ & 0.099 & 0.005 & 18.795 & 32 & 0.000 \\
\hline
\end{tabular}

\section{European hake (Merluccius merluccius Linneaus, 1758)}

A total number of 1978 European hake were caught. While $93.5 \%$ (1850) specimens retained, $6.5 \%$ (128) was escaped. Total number of specimens, captured and escaped percentage and their minimum and maximum sizes in codends are given in Table 9. Length-frequency distributions show their size ranges as 10 to $58 \mathrm{~cm}$ for all tested codends. A total of $60.5,59.6$ and $74.0 \%$ of the specimens were found above MLS in the 44T300, $44 \mathrm{~T} 150$ and $40 \mathrm{~T} 165$ codend, respectively $(20 \mathrm{~cm}$, Anonymus, 2016).

For European hake the data structure did not allow for a haul-by-haul analysis, but selection curves could be estimated for all codends based on pooled data. Selectivity estimates for pooled mean curves of European hake are given in Table 10 and Fig 3 . The pooled $\mathrm{L}_{50}$ values and $\mathrm{SR}$ in $44 \mathrm{~T} 300,44 \mathrm{~T} 150$ and $40 T 165$ were 12.8 and $4.6 \mathrm{~cm} ; 13.2$ and $4.0 \mathrm{~cm}$; and 12.1 and $1.7 \mathrm{~cm}$, respectively. The likelihood ratio test results showed that there was no significant difference between codends.

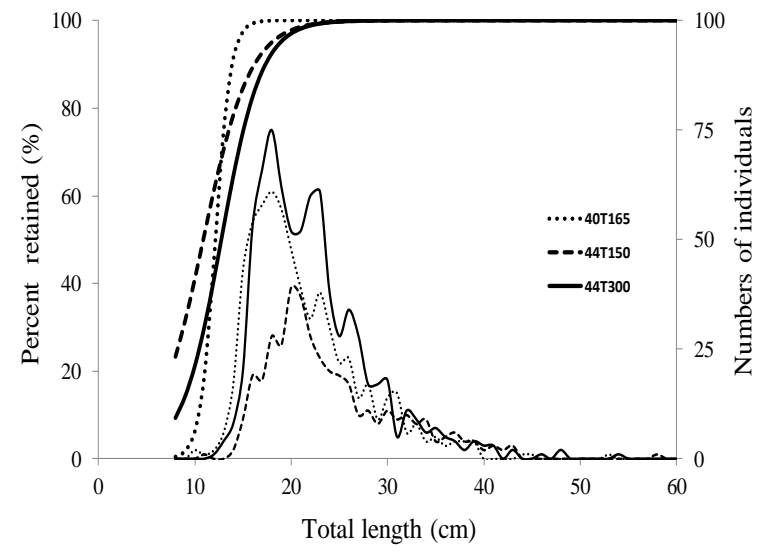

Figure 3. Mean selection curves for European hake based on pooled data with observed retention in the three codends. Thin lines correspond to length frequency in codends. Y-axis left: percentage retained for the selection curves of: a; 44T300, 44T150. Y-axis right: normalized length-frequency distribution. 
Table 9. Total number of European hake specimens, captured and escaped percentage and their minimum and maximum sizes in codends

\begin{tabular}{|c|c|c|c|c|c|c|c|c|c|c|}
\hline & \multirow{2}{*}{$\begin{array}{l}\text { Total number } \\
\text { of specimens }\end{array}$} & \multirow{2}{*}{$\begin{array}{c}\text { Captured } \\
(\%)\end{array}$} & \multicolumn{2}{|c|}{ Size ranges $(\mathrm{mm})$} & \multirow{2}{*}{$\begin{array}{l}\text { Above MLS } \\
(\%)\end{array}$} & \multirow[b]{2}{*}{ Escaped (\%) } & \multicolumn{2}{|c|}{ Size ranges $(\mathrm{mm})$} & \multirow{2}{*}{\multicolumn{2}{|c|}{$\begin{array}{l}\text { Above } \\
(\%)\end{array}$}} \\
\hline & & & Min & $\operatorname{Max}$ & & & Min & Max & & \\
\hline 44T300 & 837 & 94.0 & 12 & 48 & 59.6 & 6.0 & 15 & 19 & 0 & \\
\hline $44 \mathrm{~T} 150$ & 413 & 95.9 & 11 & 58 & 74.0 & 4.1 & 15 & 19 & 0 & \\
\hline 40T165 & 728 & 91.6 & 10 & 63 & 53.1 & 8.4 & 8 & 16 & 0 & \\
\hline
\end{tabular}

Table 10. Estimated selectivity parameters of pooled and stacked for European hake in 44T300, 44T150 and 40T165.

\begin{tabular}{|c|c|c|c|c|c|c|c|c|c|c|c|c|c|c|c|}
\hline C & & $\mathrm{L}_{50}$ & $\begin{array}{l}\text { Cl } \\
\text { low-high }\end{array}$ & SR & $\begin{array}{l}\text { Cl } \\
\text { Low-high }\end{array}$ & $v_{1}$ & $v_{2}$ & $R_{11}$ & $\mathbf{R}_{12}$ & $\mathbf{R}_{22}$ & deviance & dof & pvalue & $\mathrm{NCd}$ & $\mathrm{NCv}$ \\
\hline $44 T 300$ & $P$ & 12.8 & $12.3-13.3$ & 4.6 & $4.2-5.0$ & -6.115 & 0.479 & 0.529 & -0.463 & 0.506 & 27.64 & 31 & 0.64 & 787 & 50 \\
\hline 44T150 & $\mathbf{P}$ & 13.2 & $12.6-13.8$ & 4.0 & $3.5-4.5$ & -7.243 & 0.547 & 4.638 & -0.268 & 0.016 & 11.39 & 30 & 0.99 & 396 & 17 \\
\hline $40 \mathrm{~T} 165$ & $P$ & 12.1 & $12.1-12.2$ & 1.7 & $1.6-1.8$ & -15.385 & 1.267 & 0.076 & -0.018 & 0.051 & 6.88 & 33 & 1.00 & 667 & 61 \\
\hline
\end{tabular}

$C=$ Codends $L_{50}=$ fifty percent retention length, $C l=$ confidence interval, $S R=$ selection range; $v_{1}$ and $v_{2}$ regression parameters, $R_{11}, R_{22}$ and $R_{22}=$ variance matrix values, dof= degree of fredom

\section{DISCUSSION}

This study presents selectivity of three turned mesh codends in the Aegean Sea demersal trawl fishery. To deal with the multi-species characteristics of this fishery, the investigation focused on the main three commercial species ( $P$. longirostris, $T$. trachurus and $M$. merluccius) of this area. T90 selectivty results for hake first time determimed in the study.

There were many selectivity studies carried out with $40 \mathrm{~mm}$ diamond (Özbilgin et al., 2005, 2012; Kaykaç et al., 2009), 44 mm diamond (Aydın et al., 2009; Lucchetti, 2008; Sala et al., 2008; Aydın and Tosunoğlu, 2010) and $40 \mathrm{~mm}$ square mesh codends (Guijaro and Massuti, 2006; Lucchetti, 2008; Tosunoğlu et al., 2008b; Deval et al., 2009; Aydın and Tokaç, 2015) for three investigated species (Table 11). Some of these studies have been indicated that the selectivity of $40 \mathrm{~mm}$ diamond mesh codend are rather poor (Tokaç et al., 1998; Tokaç et al., 2004, 2010; Özbilgin et al., 2012; Aydın et al., 2011). Wienbeck et al. (2011) reported that both turning the neting direction $90^{\circ}$ and halving the number of meshes around codend circumferences had an important and possitive effect on the size selection of cod. Thus, we tested T90 codends instead of 40 and $44 \mathrm{~mm}$ diamond mesh codends. Among our three codends, the highest results were obtained from 44T150, in which both factors were applied together.

In order to increase rose shrimp selectivity, many studies have been carried out with different mesh sizes in the range of 39-60 mm (Guijaro and Massuti, 2006; Deval et al., 2006, 2009; Sala et al., 2008). However, T90 codend was used in just two studies for rose shrimp in Mediterranean. One of these studies, Aydın and Tokaç (2015) were estimated $L_{50}$ value of $14.8 \pm 0.1$ $\mathrm{mm}$ for deepwater rose shrimp with $40 \mathrm{~mm}$ turned mesh with
330 meshes on its circumference. This value was lower than our findings especialy 40T165 coded which is same mesh size with theirs. This difference could be the number of meshes around codend circumference because it is well known that decreasing number of meshes around codend, increase the L50 values. On the other hand Deval et al. (2016) investigated the selectivity of an experimental $50 \mathrm{~mm}$ diamond (T0) and $90^{\circ}$ turned mesh (T90) codends, the researchers reported $L_{50}$ value 21.4 and $22.6 \mathrm{~mm}$ for $P$. longirostris, respectively. These results are higher than our findings due to the using large meshes. $L_{50}$ value obtained in their study present a statistically significant increase with a change in the same mesh size codens from T0 to T90 for four commercial shrimp species including $P$. longirostris.

Sala et al. (2008) also reported that mesh configuration affect $L_{50}$ values of rose shrimp. Guijarro and Massuti (2006) obtained in the Western Mediterranean an increase the $L_{50}$ value for rose shrimp from 17.2 to $20.6 \mathrm{~mm}$ with a change in mesh configuration from $40 \mathrm{~mm}$ diamond to $40 \mathrm{~mm}$ square. Deval et al. (2009), using $40 \mathrm{~mm}$ square mesh codend found the same $L_{50}$ value $(18.6 \mathrm{~mm}$ ) as our results of $44 T 150$ codend. The results of this study show that $44 \mathrm{~T} 150$ codend better than the others, but none of them was adequate for the $20 \mathrm{~mm} \mathrm{MLS}$ of deepwater rose shrimp. On the other hand, there is no MLS regulation in TFR. However, Manaşılı and Avşar (2008) and Dereli (2010) studied on $50 \%$ maturity size of the $P$. longirostris, first maturity size $\left(\mathrm{CL}_{\mathrm{M} 50}\right)$ of female deepwater rose shrimps were found as $18.2 \mathrm{~mm}$ and $24.56 \mathrm{~mm}$, respectively. Dereli (2010) also estimated $\mathrm{CL}_{\mathrm{M} 50}$ of male shrimp as $12.9 \mathrm{~mm}$. Given by the results MLS regulation will be implemented by the TFR for the sustainable fishery of the rose shrimp.

Table 11. Some of the selectivity studies in Mediterranean demersal trawl fisheries

\begin{tabular}{|c|c|c|c|c|c|c|c|c|}
\hline Species & References & EsM & Area & MSh & MS & $\mathrm{CC}$ & $\mathrm{L}_{50}$ & SR \\
\hline \multirow[t]{7}{*}{ P. longirostris } & Guijaro and Massuti, (2006) & $P$ & WM & $\mathrm{S}$ & 40 & 180 & 20.2 & 2.1 \\
\hline & Aydın et al. (2009) & $\mathrm{F}$ & EM & D & 44 & 400 & 12.0 & 4.0 \\
\hline & Aydın and Tosunoğlu, (2009) & $\mathrm{F}$ & EM & $S$ & 40 & 200 & 16.7 & 6.5 \\
\hline & & $\mathrm{F}$ & EM & $\mathrm{H}$ & 40 & 200 & 17.4 & 6.2 \\
\hline & Deval et al. (2009) & $P$ & EM & S & 40 & 100 & 18.6 & 6.0 \\
\hline & & $P$ & EM & D & 44 & 200 & 16.6 & 4.4 \\
\hline & Kaykaç et. al. (2009) & $\mathrm{F}$ & EM & D & 40 & 300 & 14.5 & 5.6 \\
\hline
\end{tabular}




\begin{tabular}{|c|c|c|c|c|c|c|c|c|}
\hline & & $\mathrm{F}$ & EM & $D$ & 48 & 275 & 16.1 & 6.5 \\
\hline & \multirow{3}{*}{ Aydın et al. (2014) } & $\mathrm{F}$ & EM & $S$ & 40 & 150 & 16.3 & 4.3 \\
\hline & & $P$ & EM & $\mathrm{D}$ & 44 & *50 & 16.3 & 6.7 \\
\hline & & $P$ & EM & D & 44 & *215 & 16.8 & 6.9 \\
\hline & \multirow[t]{2}{*}{ Aydın and Tokaç, (2015) } & $\mathrm{F}$ & EM & $S$ & 40 & 165 & 15.5 & 5.4 \\
\hline & & $\mathrm{F}$ & EM & T90 & 40 & 330 & 14.8 & 7.4 \\
\hline & \multirow[t]{2}{*}{ Deval et al. (2016) } & $\mathrm{F}$ & EM & $\mathrm{D}$ & 50 & 300 & 21.4 & 9.3 \\
\hline & & $\mathrm{F}$ & EM & T90 & 50 & 300 & 22.6 & 8.3 \\
\hline & \multirow[t]{3}{*}{ Present study } & $\mathrm{F}$ & EM & T90 & 44 & 300 & 17.5 & 5.7 \\
\hline & & $\mathrm{F}$ & EM & T90 & 44 & 150 & 18.6 & 5.1 \\
\hline & & $\mathrm{F}$ & EM & T90 & 40 & 165 & 17.1 & 6.0 \\
\hline \multirow[t]{13}{*}{ T.trachurus } & Tosunoğlu et al. (2008a) & $\mathrm{F}$ & EM & $\mathrm{D}$ & 50 & 400 & 15.6 & 5.5 \\
\hline & \multirow[t]{3}{*}{ Aydın and Tosunoğlu, (2010) } & $\mathrm{F}$ & EM & $D$ & 44 & 400 & 14.7 & 4.6 \\
\hline & & $\mathrm{F}$ & EM & $S$ & 40 & 200 & 15.9 & 5.6 \\
\hline & & $\mathrm{F}$ & EM & $\mathrm{H}$ & 40 & 200 & 17.1 & 5.0 \\
\hline & \multirow[t]{2}{*}{ Aydın et al. (2014) } & $\mathrm{F}$ & EM & D & 44 & $* 50$ & 16.3 & 5.5 \\
\hline & & $\mathrm{F}$ & EM & D & 44 & *215 & 16.7 & 6.2 \\
\hline & \multirow[t]{4}{*}{ Dereli and Aydın, (2016) } & $\mathrm{F}$ & EM & D & 44 & 300 & 16.2 & 3.1 \\
\hline & & $\mathrm{F}$ & EM & $\mathrm{D}$ & 50 & 264 & 14.2 & 4.2 \\
\hline & & $\mathrm{F}$ & EM & $S$ & 40 & 165 & 15.3 & 3.2 \\
\hline & & $\mathrm{F}$ & EM & T90 & 40 & 330 & 17.1 & 2.1 \\
\hline & \multirow[t]{3}{*}{ Present study } & $\mathrm{F}$ & EM & T90 & 44 & 300 & 16.4 & 5.7 \\
\hline & & $\mathrm{F}$ & EM & T90 & 44 & 150 & 17.1 & 5.1 \\
\hline & & $\mathrm{F}$ & EM & T90 & 40 & 165 & 14.8 & 6.0 \\
\hline \multirow[t]{23}{*}{ M. merluccius } & Özbilgin et al. (2005) & $\mathrm{F}$ & EM & $\mathrm{D}$ & 40 & 100 & 14.3 & 3.4 \\
\hline & \multirow[t]{2}{*}{ Bahamon et al. (2006) } & $P$ & NWM & $D$ & 40 & 230 & 10.1 & 3.1 \\
\hline & & $P$ & NWM & $S$ & 40 & 140 & 16.0 & 3.2 \\
\hline & \multirow[t]{2}{*}{ Guijaro and Massuti, (2006) } & $P$ & WM & D & 40 & & 11.6 & 3.1 \\
\hline & & $P$ & WM & $S$ & 40 & 180 & 15.3 & 2.2 \\
\hline & Lucchetti, (2008) & $\mathrm{F}$ & $\mathrm{CM}$ & $S$ & 40 & 310 & 13.0 & 3.7 \\
\hline & Tosunoğlu et al. (2008a) & $\mathrm{F}$ & EM & $D$ & 50 & 400 & 11.4 & 4.1 \\
\hline & \multirow[t]{3}{*}{ Aydın and Tosunoğlu, (2010) } & $\mathrm{F}$ & EM & $\mathrm{D}$ & 44 & 400 & 10.4 & 3.1 \\
\hline & & $\mathrm{F}$ & EM & $S$ & 40 & 200 & 14.4 & 4.8 \\
\hline & & $\mathrm{F}$ & EM & $\mathrm{H}$ & 40 & 200 & 11.0 & 4.3 \\
\hline & \multirow{2}{*}{ Sala and Lucchetti, (2011) } & $\mathrm{F}$ & $\mathrm{CM}$ & $\mathrm{D}$ & 48 & 280 & 11.5 & 5.6 \\
\hline & & $\mathrm{F}$ & $\mathrm{CM}$ & $D$ & 56 & 240 & 16.3 & 7.8 \\
\hline & \multirow[t]{3}{*}{ Özbilgin et al. (2012) } & $\mathrm{F}$ & EM & $D$ & 40 & 300 & 10.5 & 3.8 \\
\hline & & $\mathrm{F}$ & EM & $\mathrm{D}$ & 48 & 275 & 12.8 & 3.7 \\
\hline & & $\mathrm{F}$ & EM & $S$ & 40 & 150 & 15.2 & 4.7 \\
\hline & \multirow[t]{2}{*}{ Aydın et al. (2014) } & $P$ & EM & $D$ & 44 & *50 & 9.7 & 7.2 \\
\hline & & $P$ & EM & $D$ & 44 & *215 & 10.4 & 4.2 \\
\hline & \multirow[t]{3}{*}{ Dereli and Aydın, (2016) } & $\mathrm{F}$ & EM & D & 44 & 300 & 12.3 & 1.6 \\
\hline & & $\mathrm{F}$ & EM & $\mathrm{D}$ & 50 & 264 & 14.4 & 6.3 \\
\hline & & $\mathrm{F}$ & EM & $S$ & 40 & 165 & 14.3 & 3.4 \\
\hline & \multirow[t]{3}{*}{ Present study } & $\mathbf{P}$ & EM & T90 & 44 & 300 & 12.8 & 4.6 \\
\hline & & $\mathbf{P}$ & EM & T90 & 44 & 150 & 13.2 & 4.0 \\
\hline & & $\mathbf{P}$ & EM & T90 & 40 & 165 & 12.1 & 1.7 \\
\hline
\end{tabular}

NWM, North western-;WM,western-;EM, eastern-; CM, central Mediterranean; EsM, method for estimation of selectivity (F, Fryer's; P, pooled); MSh, mesh shape (D, diamond-; S, square-; H,hexagonal-;T90, 90 \% turned mesh); MS, mesh size; $L_{50}$, mean value of retention length at $50 \%$; SR, selection range

To the best of our knowledge, there is only one study about T90 turned mesh codends for horse mackerel in the Aegean Sea (Dereli and Aydın, 2016). Among three different mesh configurations, the highest $L_{50}$ value $(17.1 \mathrm{~cm})$ for horse mackerel was obtained from T90 codend by researchers. Although their T90 codend has both smaller mesh size and wider codend than our $44 T 300$ codend, L50 values were obtained equal. Our results of $44 \mathrm{~mm}$ turned mesh codends are higher than $44 \mathrm{~mm}$ diamond mesh codends given by same fishing grounds (Aydın and Tosunoğlu, 2010). They are estimated a $L_{50}$ value of $14.7 \mathrm{~cm}$ for the $44 \mathrm{~mm}$ diamond mesh with 400 meshes around codend circumference. This value $(14.7 \mathrm{~cm})$ is close to just our $40 \mathrm{~mm}$ turned mesh codend. This closeness may be resulted of using narrower codend in our study according to the other. Morphological and behavioural characters may also explain the higher $L_{50}$ values for $T$. trachurus. Tosunoğlu et al., (2008a) indicate that most horse mackerel individuals showed continuous stronger swimming ability in the belly section of the trawl until the trawl was hauled up. For this reason, $L_{50}$ values of the codend for mackerel is $20 \%$ higher than the MLS $(13 \mathrm{~cm})$, thus allowing for the escapement of most the juveniles.

Studies that carried out different mesh shape, size and material, ranging from 28 to $70 \mathrm{~mm}$ mesh size in Mediterranean (Özbilgin et al., 2005, 2012; Guijaro and Massuti, 2006; Lucchetti, 2008; Tosunoğlu et al., 2008a; Aydın and Tosunoğlu, 2010) for hake. However, due to the differences in the mesh size and material, mesh configuration and number of meshes at the codend circumference, results of most of these studies are not comparable to those found in the existing findings (Tosunoğlu et al., 2008a; Sala and Lucchetti, 2010). Their 
results show that the use of larger diamond mesh codend increases the $L_{50}$ values for $M$. merluccius. Furthermore, the analysis of selectivity presented that square mesh codend positively affected $L_{50}$ of hake; this codend increased the size of escapement in the Mediterranean (Guijarro and Massuti, 2006; Ordines et al., 2006; Lucchetti, 2008; Aydın and Tosunoğulu, 2010; Özbilgin et al., 2012; Dereli and Aydın, 2016). But none of them mentioned T90 codends. The present study for the first time presents selectivty parameters of T90 for hake. The results of $L_{50}(13.2 \mathrm{~cm})$ value of the $44 T 150$ codend is higher than the values for 44T150 and 40T165 codends. However, these $L_{50}$ values are still very low compared to the 20 $\mathrm{mm}$ MLS (Anonymous, 2016). On the otherhand, Our $40 \mathrm{~mm}$ turned mesh size codend (40T165) selectivity results are higher than those reported by Bahamon et al. (2006), Guijarro and Massuti (2006) and Tosunoğlu et al. (2008a) who determined $L_{50}$ values for $40 \mathrm{~mm}$ nominal mesh sizes as $10.1 \mathrm{~cm}, 11.6 \mathrm{~cm}$ and $11.4 \mathrm{~cm}$, respectively. From the Table 11, no investigations were adequate release the under $20 \mathrm{~cm}$ individuals.

Sala and Lucchetti (2011) reported that the codend circumference plays a role which is as important as mesh size for M. merluccius, M. barbatus and Alloteuthis media. An increase in mesh size from $48 \mathrm{~mm}$ to $56 \mathrm{~mm}$ could be made futile by an increase in codend circumference of only $13-17 \%$. Tosunoğlu et al. (2008a) and Sala and Lucchetti (2011) also found that selectivity increases with larger mesh size but also decreases with wider codends. These results showed that it is not sufficient to increase selectivity in multi-species fisheries only by setting a minimum mesh size, account must also be taken of the effects of the other factors such as the codend circumference, mesh configuration.

\section{REFERENCES}

Anonymous 2006. Council Regulation (EC 1967/2006) concerning management measures for the sustainable exploitation of fishery resources in the Mediterranean Sea, amending regulation (EEC) No 2847/93 and repealing Regulation (EC) No 1626/94. Off. J.E.U. 409, 75 p

Anonymous (2012). Notification 3/1 Regulating Commercial Fishing (in Turkish). Republic of Turkey, Minister of Agriculture and Rural Affair General Directorate of Conservation and Inspection, Ankara, 112 pp.

Anonymous (2016). Notification 4/1 Regulating Commercial Fishing (in Turkish). Republic of Turkey, Minister of Agriculture and Rural Affair, General Directorate of Conservation and Inspection, Ankara, 68 pp.

Arkley, K. (2008). Reducing drag in towed fishing gear - fishing trials to evaluate the performance of a trawl constructed from T90 ('turned mesh') netting. Final Report, SR595, $37 \mathrm{pp}$.

Aydın, C., \& Tokaç, A. (2015). Selectivity of $40 \mathrm{~mm}$ square and $90^{\circ}$ turnedmesh codend for the deepwater rose shrimp, Parapenaeus longirostris (Crustacea) and greater forkbeard, Phycis blennoides (Actinopterygii: Gadiformes: Phycidae), in the Eastern Mediterranean. Acta Ichthyologica et Piscatoria, 45 (4), 353-362. DOI: 10.3750/AIP2015.45.4.03

Aydın, C., Tokaç, A., Ulaş, A., Maktay, B., \& Şensurat, T. (2011). Selectivity of $40 \mathrm{~mm}$ square and $50 \mathrm{~mm}$ diamond mesh codends for five species in the Eastern Mediterranean demersal trawl fishery. African Journal of Biotechnology, 10(25), 5037-5047. DOI: 10.5897/AJB11.082

Aydın, C., \& Tosunoğlu, Z. (2009). Selectivity of square and hexagonal mesh codends for the deep water rose shrimp, Parapenaus longirostris (Lucas,
In conclusion, the change from 300 to 150 number of meshes around the codend circumference, increase $L_{50}$ from 17.5 to $18.6 \mathrm{~mm}(6.3 \%)$ for deepwater rose shrimp, from 16.4

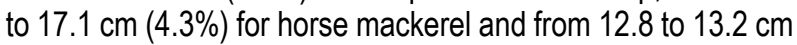
(3.1\%) for hake. However, these values no adequate for the MLS of deepwater rose shrimp and European hake. Therefore, an option of reducing the number of meshes around codend circumference with a larger turned mesh should be researched in future studies for obtaining optimal $50 \%$ retention length values. It is known that any efforts such as using square mesh, turned mesh codends or halved the number of meshes in the codend to release immatures of some species, is probably to cause the loss of marketable sizes of other species in the Mediterranean. This economical loss of revenues is the essential concern of fishermen that prevents them using alternative codend designs, though such designs are technically applicable. Consequently, the losses of income caused by using T90 mesh codend and halving the number of meshes around codend circumference should also be investigated in future studies.

\section{ACKNOWLEDGEMENTS}

The authors would like to thank the captain Hüseyin TÜRKER and crew of the commercial trawler "Efsane G". We would also like to thank George Masser for helpful language revision of the manuscript. We are grateful to the journal editor and two anonymous referees for their valuable contributions. This work is derived from the Phd dissertation of the first author.

1846) (Decapoda, Penaidae) in the Aegean Sea. Crustaceana, 82(1), 8998. DOI: $10.1163 / 156854008 \times 363704$

Aydın, C., \& Tosunoğlu, Z. (2010). Selectivity of diamond, square and hexagonal mesh codends for Atlantic horse mackerel Trachurus trachurus, European hake Merluccius merluccius, and greater forkbeard Phycis blennoides in the eastern Mediterranean. Journal of Applied Ichthyology, 26(1), 71-77. DOI: 10.1111/j.1439-0426.2009.01376.x

Aydın, C., Tosunoğlu, Z., \& Özbilgìn, H. (2009). Selectivity of double and single codends for the deep-water rose shrimp, Parapenaeus longirostris (Lucas, 1846) (Decapoda, Penaeidae) in the Aegean Sea trawl fishery. Crustaceana, 82(2), 233-240. DOI: 10.1163/156854008x380264

Bahamon, N., Sarda, F., \& Suuronen, P. (2006). Improvement of trawl selectivity in the NW Mediterranean demersal fishery by using a $40 \mathrm{~mm}$ square mesh codend. Fisheries Research, 81(1), 15-25. DOI: 10.1016/j.fishres.2006.05.020

Broadhurst, M. K., \& Kennelly, S. J. (1996). Effects of the circumference of codends and a new design of square-mesh panel in reducing unwanted by-catch in the New South Wales oceanic prawn-trawl fishery, Australia. Fisheries Research, 27(4), 203-214. DOI: 10.1016/0165-7836(95)00469-6

Broadhurst, M. K., Millar, R. B., Kennelly, S. J., Macbeth, W. G., Young, D. J., \& Gray, C. A. (2004). Selectivity of conventional diamond-and novel square-mesh codends in an Australian estuarine penaeid-trawl 
fishery. Fisheries Research, 67(2), 183-194. DOI: 10.1016/j.fishres.2003.09.043

Campos, A., Fonseca, P., \& Henriques, V. (2003). Size selectivity for four fish species of the deep groundfish assemblage off the Portuguese southwest coast: evidence of mesh size, mesh configuration and cod end catch effects. Fisheries research, 63(2), 213-233. DOI: 10.1016/S0165-7836(03)00060-2

ConStat (1995). CC selectivity. Gronspaettevej 10, DK-9800 Hjorring Denmark.

Dereli, H. (2010). Sığacık Körfezi'nde dip troll ağları ile yakalan derinsu pembe karidesi (Parapenaeus longirostris Lucas, 1846)'nin bazı biyolojik ve populasyon özellikleri. Ege Üniversitesi Fen Bilimleri Enstitüsü, Doktora Tezi, İzmir (in Turkish)

Dereli, H., \& Aydın, C. (2016). Selectivity of Commercial and Alternative Codends for Four Species in the Eastern Mediterranean Demersal Traw Fishery. Turkish Journal of Fisheries and Aquatic Sciences, 16(4), 971 992. DOI: 10.4194/1303-2712-v16_4_25

Deval, M. C., Bök, T., Ateş, C., \& Özbilgin, H. (2006). Selectivity of PE and PA material cod ends for rose shrimp (Parapenaeus longirostris) in Turkish twin rigged beam trawl fishery. Fisheries Research, 81, 72-79.

Deval, M. C., Bök, T., Ateş, C., Ulutürk, T., \& Tosunoğlu, Z. (2009). Comparison of the size selectivity of diamond (PA) and square (PE) mesh codends fo deepwater crustacean species in the Antalya Bay, Eastern Mediterranean. Journal of Applied Ichthyology, 25, 372-380.

Deval, M.C., Özgen, G., \& Özbilgin, H. (2016). Selectivity of 50 mm T0 and T90 codends for commercial shrimp species in the Turkish deepwater traw fishery, Eastern Mediterranean. Journal of Applied Ichtyology, 32 (6) 1041-1057. DOI: 10.1111/jai.13128

Eryaşar, A.R. (2014). Mersin Körfezi dip trollerinde ticari ve alternative torbaların seçiciliği ve seçicilik ızgaralarında balık davranışları. Mersin Üniversitesi Fen Bilimleri Enstitüsü Doktora Tezi, Mersin (in Turkish).

EUROPEAN UNION, 2005. Council Regulation No. 2187/2005 of 21 December 2005 , for the conservation of fishery resources through technical measures in the Baltic Sea, the Belts and the Sound, amending Regulation (EC) No. 1434/98 and repealing Regulation (EC) No. 88/98. Official Journal of the European Union L., 349/1.

Fryer, R.J. (1991). A model of between-haul variation in selectivity. ICES Journal of Marine Science, 48(3), 281-290. DOI: 10.1093/icesjms/48.3.281

Gorman, D., \& Dixon, C. (2015). Reducing discards in a temperate prawn traw fishery: a collaborative approach to bycatch research in South Australia. ICES Journal of Marine Science, 72(9), 2609-2617. DOI: 10.1093/icesjms/fsv147

Guijarro, B., \& Massutí, E. (2006). Selectivity of diamond-and square-mesh codends in the deepwater crustacean trawl fishery off the Balearic Islands (western Mediterranean). ICES Journal of Marine Science, 63(1), 52-67. DOI: 10.1016/j.icesjms.2005.08.011

Hansen, U.J. (2004). Performance of a trawl made from $90^{\circ}$ turned netting (T90) compared with that of traditional codends. Gydynia: ICES Fishing Technology and Fish Behaviour Working Group Meeting.

Herrmann, B., Priour, D., \& Krag, L. A. (2007). Simulation-based study of the combined effect on cod-end size selection of turning meshes by 90 and reducing the number of meshes in the circumference for round fish. Fisheries Research, 84(2), 222-232. DOI: 10.1016/j.fishres.2006.10.020

Herrmann, B., Wienbeck, H., Moderhak, W., Stepputtis, D., \& Krag, L. A (2013). The influence of twine thickness, twine number and netting orientation on codend selectivity. Fisheries research, 145, 22-36. DOI: 10.1016/j.fishres.2013.03.002

İlkyaz, A. T., Şensurat, T., Dereli, H., \& Aydın, C. (2017). Codends Selectivity for Bogue (Boops boops L., 1758) in the Eastern Mediterranean Demersal Trawl Fishery. Turkish Journal of Fisheries and Aquatic Sciences, 17(4), 673-680. DOI: 10.4194/1303-2712-v17_4_03
Kaykaç, M. H. (2005). Geleneksel dip trol ağında torba ağ göz açılımını arttırmaya yönelik çalışmalar. Ege Üniversitesi Fen Bilimleri Enstitüsü Doktora Tezi, $128 \mathrm{~s}$, Izmir (in Turkish).

Kaykaç, H., Tokaç, A., \& Özbilgin, H. (2009). Selectivity of commercial, larger mesh and square mesh trawl codends for deep water rose shrimp Parapenaeus longirostris (Lucas, 1846) in the Aegean Sea. Scientia Marina, 73(3), 597-604

Lök, A., Tokac, A., Tosunoǧlu, Z., Metin, C., \& Ferro, R. S. T. (1997). The effects of different cod-end design on bottom trawl selectivity in Turkish fisheries of the Aegean Sea. Fisheries Research, 32(2), 149-156. DOI: 10.1016/S0165-7836(97)00048-9

Lucchetti, A. (2008). Comparison of diamond- and square-mesh codends in the hake (Merluccius merluccius L. 1758) trawl fishery of the Adriatic Sea (central Mediterranean). Sci. Mar., 72: 451-460.

DOI: 10.3989/scimar.2008.72n3451

Madsen, N. (2007). Selectivity of fishing gears used in the Baltic Sea cod fishery. Reviews in Fish Biology and Fisheries, 17(4), 517-544 DOI: 10.1007/s11160-007-9053-y

Madsen, N., Hansen, K., \& Madsen, N. A. (2015). Behavior of different trawl codend concepts. Ocean Engineering, 108, 571-577. DOI: 10.1016/j.oceaneng.2015.08.047

Madsen, N., Herrmann, B., Frandsen, R. P., \& Krag, L. A. (2012). Comparing selectivity of a standard and turned mesh T90 codend during towing and haul-back. Aquatic Living Resources, 25(3), 231-240. DOI: 10.1051/alr/2012021

Manaşılı, M., \& Avşar, D. (2008). Reproductive biology of female Parapenaeus longirostris (Lucas, 1846) (Decapoda, Caridea) in Babadillimanı Bight in the northeastern Mediterranean. Crustaceana, 81: 289-298.

McCullagh, P., \& Nelder, J. A. (1989). Generalized linear models, 2nd edn. London: Chapman and Hall. 511 pp.

Moderhak, W. (1997). Determination of selectivity of cod codends made of turned through $90^{\circ}$. Bulletin of the Sea Fisheries Institute, Gdynia, 1(140):414

Ordines, F., Massutí, E., Guijarro, B., \& Mas, R. (2006). Diamond vs. square mesh codend in a multi-species trawl fishery of the western Mediterranean: effects on catch composition, yield, size selectivity and discards. Aquatic Living Resources, 19(4), 329-338.

Özbilgin, H., Tokaç, A., \& Kaykaç, H. (2012). Selectivity of commercial compared to larger mesh and square mesh trawl codends for four fish species in the Aegean Sea. Journal of Applied Ichthyology, 28(1), 51-59. DOI: 10.1111/j.1439-0426.2011.01916.x

Özbilgin, H., Tosunoğlu, Z., Aydın, C., Kaykaç, H., \& Tokac, A. (2005) Selectivity of standard, narrow and square mesh panel trawl codends for hake (Merluccius merluccius) and poor cod (Trisopterus minutus capelanus). Turkish Journal of Veterinary and Animal Sciences, 29(4), 967-973.

Reeves, S. A., Armstrong, D. W., Fryer, R. J., \& Coull, K. A. (1992). The effects of mesh size, cod-end extension length and cod-end diameter on the selectivity of Scottish trawls and seines. ICES Journal of Marine Science, 49(3), 279-288. DOI: 10.1093/icesjms/49.3.279

Sala, A., \& Luchetti, A. (2010). The effect of mesh configuration and codend circumference on selectivity in the Mediterranean trawl Nephrops fishery. Fisheries Research, 103: 63-72.

Sala, A., \& Lucchetti, A. (2011). Effect of mesh size and codend circumference on selectivity in the Mediterranean demersal trawl fisheries. Fisheries Research, 110(2), 252-258. DOI: 10.1016/j.fishres.2011.04.012

Sala, A., Lucchetti, A., Piccinetti, C., \& Ferreti, M. (2008). Size selection by diamond and square-mesh codends in multi-species Mediterranean demersal trawl fisheries. Fisheries Research, 93: 8-21. DOI: 10.1016/j.fishres.2008.02.003

Stepputtis, D., Santos, J., Herrmann, B., \& Mieske, B. (2016). Broadening the horizon of size selectivity in trawl gears. Fisheries Research, 184, 18-25. DOI: 10.1016/j.fishres.2015.08.030

Stewart, P. A. (2002). A review of studies of fishing gear selectivity in the Mediterranean. FAO COPEMED Report No. 9, Rome, Italy, 57 pp. 
Şensurat, T. (2015). Dip trol ağlarında Derinsu pembe karidesi (Parapenaus longirostris), bakalyaro (Merluccius merluccius) ve istavrit (Trachurus trachurus)'in $90^{\circ}$ döndürülmüş torba seçiciliği. Atatürk Üniversitesi Fen Bilimleri Enstitüsü, Doktora Tezi, Erzurum (in Turkish).

Tokaç, A., Herrmann, B., Aydın, C., Kaykac, H., Ünlüler, A., \& Gökce, G. (2014) Predictive models and comparison of the selectivity of standard (T0) and turned mesh (T90) codends for three species in the Eastern Mediterranean. Fisheries Research, 150, 76-88. DOI: 10.1016/j.fishres.2013.10.015

Tokaç, A., Lök, A., Tosunoğlu, Z., Metin, C., \& Ferro, R.S.T. (1998). Cod-end selectivities of a modified bottom trawl for three fish species in the Aegean Sea. Fisheries Research, 39(1), 17-31. DOI: 10.1016/S0165-7836(98)00172-6.

Tokaç, A., Özbilgin, H., \& Kaykaç, H. (2010). Selectivity of conventional and alternative codend design for five fish species in the Aegean Sea. Journal of Applied Ichthyology, 26(3), 403-409. DOI: $10.1111 /$ j.1439-0426.2009.01379.x

Tokaç, A., Özbilgin, H., \& Tosunoğlu, Z. (2004). Effect of PA and PE material on codend selectivity in Turkish bottom trawl. Fisheries Research, 67(3), 317-327. DOI: 10.1016/j.fishres.2003.10.001

Tosunoğlu, Z., Aydın, C., \& Özaydın, O. (2008). Selectivity of a 50-mm diamond mesh knotless polyethylene codend for commercially important fish species in the Aegean Sea. Journal of Applied Ichthyology, 24(3), 311315. DOI: 10.1111/j.1439-0426.2008.01067.x

Tosunoğlu, Z., Aydın, C., Saygı, H., Soykan O., Dereli, H., Leblebici, S., Hepkafadar, O., \&Maktay, B. (2008b). Dip Trol Ağlarında farklı torba tasarımları ile ticari türlerde boy seçiciliğinin geliştirilmesi. Ege Üniversitesi BAP Proje Final Raporu, 2007/BIL/004, İzmir (In Turkish)

Tosunoğlu, Z., Doğanyılmaz Özbilgin, Y., \& Özbilgin, H. (2003). Body shape and trawl cod end selectivity for nine commercial fish species. Journal of Marine Biology Association of the UK, 83, 1309-1313. DOI: $10.1017 /$ S0025315403008737

Wienbeck, H., Herrmann, B., Feekings, J. P., Stepputtis, D., \& Moderhak, W. (2014). A comparative analysis of legislated and modified Baltic Sea trawl codends for simultaneously improving the size selection of cod (Gadus morhua) and plaice (Pleuronectes platessa). Fisheries Research, 150, 28 37. DOI: 10.1016/j.fishres.2013.10.007

Wienbeck, H., Herrmann, B., Moderhak, W., \& Stepputtis, D. (2011). Effect of netting direction and number of meshes around on size selection in the codend for Baltic cod (Gadus morhua). Fisheries Research, 109(1), 8088. DOI: 10.1016/j.fishres.2011.01.019

Wileman, D., Ferro, R.S.T. Fonteyne, R., \& Millar, R. B. (1996). Manual of methods of measuring the selectivity of towed fishing gears. ICES Cooperative Research Report No. 215, 126 pp. 